En 1835, Gustave Coriolis dériva de ses équations

les forces qui agissent sur les corps en déplacement dans un système en rotation. Son travail trouva l'inspiration dans l'étude des machines tournantes - nous étions alors en pleine révolution industrielle. Pourtant, le premier système en rotation qui s'impose à nous naturellement, et de la même façon à travers les époques, est bien la Terre elle-même. II n'est donc pas étonnant que la question de I'influence de la rotation de la Terre sur les mouvements des corps ait été abordée par d'autres scientifiques, bien avant Coriolis.

\section{Nous proposons ici un} récapitulatif de ces travaux précurseurs.

(1) Hauteur de la Torre degli Asinelli à Bologne. (1) Hauteur de la Torre degli Asinelli à Bologne,
depuis laquelle des expériences de chute libre furent réalisées à l'époque.

(2) Borelli confondit également conservation de la vitesse et conservation du moment cinétique dans son calcul sur la chute des corps.

Visualisation de tourbillons par fluorescence sur la plate-forme Coriolis, dans le cadre de l'étude de l'interaction entre deux cyclones intenses, connue sous le nom d'effet Fujiwara. Expériences menées par Jan-Bert Flor, LEGl, 2005.

\title{
Petite histoire de la force de Coriolis
}

Theo Gerkema ${ }^{(a)}$ et Louis Gostiaux ${ }^{(\mathbf{b})}$ (louis.gostiaux@legi.grenoble-inp.fr)

(a) NIOZ - Royal Netherlands Institute for Sea Research, Texel, Pays-Bas

(b) LEGI, Plate-forme Coriolis, B.P. 53, 38041 Grenoble Cedex 9

Les premières idées concernant l'influence de la rotation de la Terre sur les mouvements des corps ont été introduites lors du débat sur l'existence même de cette rotation. Ceci était inévitable, car la physique aristotélicienne fournissait un argument de poids contre son existence : tout ce qui s'élève dans le ciel, comme par exemple les oiseaux ou les nuages, ne partage plus un lien solide avec la Terre, et doit, pour un observateur resté sur celle-ci, se déplacer vers l'Ouest avec une grande vitesse. Ce n'est évidemment pas ce que l'on observe, et il semblait donc s'ensuivre que la Terre ne tourne pas! Pour dépasser cet argument-ci, il fallut introduire un autre principe d'inertie. Il fut fourni par Galilée, qui affirma que les objets persistent dans leur mouvement horizontal, ce qui veut dire chez lui : ils continuent dans leur trajectoire circulaire, et tournent donc avec la Terre.

\section{La chute des corps}

Giovanni Borelli, membre de l'Accademia del Cimento de Florence, tira en 1668 les conséquences de ce principe pour la chute des corps [1]. Sur la Terre tournante, le sommet d'un édifice décrit dans l'espace un cercle de plus grand rayon que son pied, et a donc une vitesse plus grande vers l'Est. Or, Borelli supposait qu'un corps qui tombe conserve son surplus de vitesse, et qu'il va donc s'écarter un peu à l'Est par rapport à la verticale. Borelli calcula la valeur de cette déviation : pour une tour d'une hauteur de 71 mètres (240 pieds $\left.{ }^{(1)}\right)$, placée sur l'équateur, il trouva une valeur de $2 \mathrm{~cm}$. Nous n'entrerons pas dans les détails de son raisonnement, qui a d'ailleurs quelques faiblesses. Ceci ne l'empêcha pourtant pas de trouver la bonne direction et le bon ordre de grandeur.

Une dizaine d'années plus tard, dans une lettre à Hooke, Newton évoqua lui aussi la possibilité d'une preuve expérimentale de la rotation de la Terre par la déviation vers l'Est de la chute libre. Hooke fit cette expérience en effet plusieurs fois, et crut en avoir fourni la preuve, ce qui est pourtant très douteux car il faut bien des précautions pour faire l'expérience d'une manière suffisament précise.

Il se passa plus de cent ans avant qu'une avancée importante sur la question eût lieu. En 1803, Laplace et Gauss derivèrent 
(indépendamment l'un de l'autre) l'expression correcte pour la déviation (d) vers l'Est que subit un corps en chute libre :

\section{$d=(2 / 3) \Omega \cos \varphi\left(2 h^{3} / g\right)^{1 / 2}$}

Ici $\Omega$ est la vitesse angulaire de la Terre $\left(7,292 \times 10^{-5} \mathrm{rad} / \mathrm{s}\right)$ et $\varphi$ la latitude $; g$ est l'accélération due à la gravité, et $h$ l'altitude d'où tombe le corps [2].

À la même époque, des expériences furent effectuées dans lesquelles on a soigneusement essayé de minimiser toutes les perturbations. Les plus connues sont celles réalisées par Ferdinand Reich dans un puits de mine à Freiberg (Saxe), sur une profondeur de 158,5 m [2]. Sur un total de 106 expériences il trouva une déviation moyenne vers l'Est de $2,8 \mathrm{~cm}$, ce qui est égal à la valeur théorique. La dispersion des valeurs est pourtant grande (fig. 1) : maintes fois, la boule tomba même vers l'Ouest !

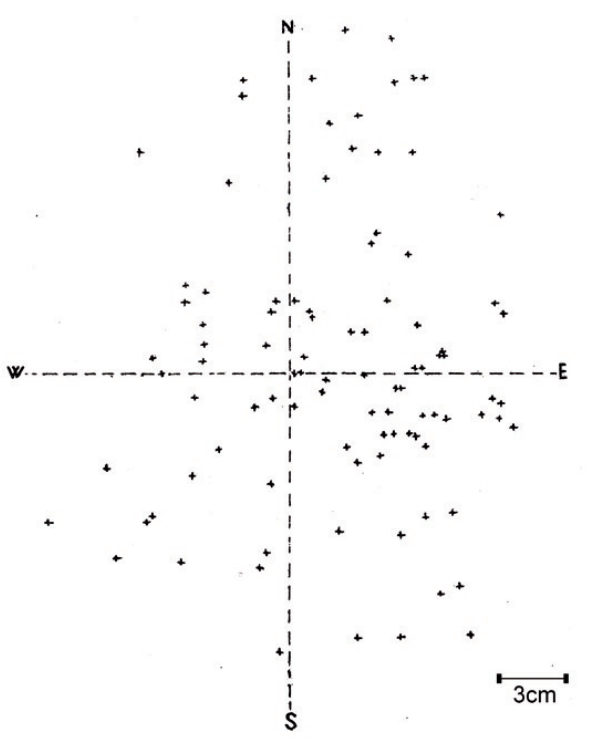

1. Le résultat d'une centaine d'expériences de chute libre dans un puits de mine, réalisées par Reich en 1831. [Figure empruntée à Hagen (1911)].

\section{Les alizés}

Dans les régions équatoriales règnent les alizés : des vents stables qui soufflent vers le Sud-Ouest dans l'hémisphère Nord, et vers le Nord-Ouest dans l'hémisphère Sud (fig. 2). Ils jouèrent un rôle important pour la découverte du continent américain, et furent naturellement l'objet de nombreuses études depuis cette époque. Il s'agissait notamment de comprendre les deux composantes de ces vents : leur convergence vers l'équateur et leur mouvement général vers l'Ouest.

L'idée que les alizés puissent être influencés par la rotation de la Terre fut déjà évoquée au début du $17^{\mathrm{e}}$ siècle : on pensait que l'air près de l'équateur ne pourrait pas suivre la grande vitesse de la Terre, ce qui expliquerait pourquoi les vents y soufflent vers l'Ouest (l'influence de la pensée aristotélicienne est ici encore évidente).

Quant à la convergence vers l'équateur, elle fut expliqué par Mariotte en 1686 : à l'équateur l'air est plus chauffé, ce qui le fait monter ; le déficit ainsi créé doit être rempli par l'air venant des latitudes plus hautes et plus basses, ce qui donne alors un vent vers l'équateur [3].

En 1735, George Hadley relia cette convergence au mouvement vers l'Ouest : il interpréta les alizés comme un vent dirigé vers l'équateur mais qui est dévié vers l'Ouest par la rotation de la Terre. Ainsi il illumina le vrai rôle de la rotation de la Terre : celle-ci ne crée pas le vent; elle ne fait que dévier un vent qui existe déjà. De plus, Hadley proposa un mécanisme pour cette déviation : une particule se déplaçant vers l'équateur vient d'un parallèle plus petit que celui où elle va; ce déficit dans la vitesse vers l'Est explique selon lui la déviation vers l'Ouest. En réalité, il faudrait appliquer le principe de la conservation du moment cinétique (ou « principe des aires »), et non pas celui de la conservation des vitesses linéaires. Cette correction fait doubler la déviation [4]. ${ }^{(2)}$

\section{Laplace et l'approximation " traditionnelle »}

Une grande avancée théorique fut apportée par Laplace dans son étude des marées [4]. Il y étudia la propagation de celles-ci dans un océan hypothétique qui recouvrirait la Terre entière. Afin de pouvoir analyser ce problème, il fallait tout d'abord établir l'expression des forces qui s'exercent dans un système tournant avec la Terre. Laplace fut le premier à le faire d'une manière exacte et complète. Son analyse montre en particulier ce qui se passe lorsque des particules se déplacent; elles subissent alors une force qui les dévie dans une direction perpendiculaire à leur propre vitesse. Dans le système de coordonnées géographiques habituel, Laplace montra qu'il apparaît quatre termes de ce type dans les équations régissant la quantité de mouvement (voir encadré ci-dessous).

Son analyse contient cependant encore un autre point important. Laplace remarqua que l'océan n'est qu'une couche de très faible épaisseur comparée au rayon de la Terre. Cette contrainte géométrique implique que les mouvements à grande échelle sont forcément quasi horizontaux ; ceci rend les termes (2) et (4) relativement moins importants. Laplace les négligera dans la suite de son analyse et ne tiendra compte que des termes (1) et (3). Depuis, cette approximation a été faite quasi universellement dans les études en dynamique des fluides géophysiques, et est donc, à juste titre, connue comme l'approximation " traditionnelle ». (Des études récentes montrent cependant qu'elle est discutable dans certains cas [6].)

\section{- Les 4 termes régissant la déflection due à la rotation de la Terre}

- une vitesse vers l'Est $(u)$ donne (1) une force vers le Sud (dont l'expression est $-2 \Omega \sin (\varphi) u)$, ainsi que (2) une force verticale vers le haut $(2 \Omega \cos (\varphi) u)$;

- une vitesse vers le Nord $(v)$ donne (3) une force vers l'Est $(2 \Omega \sin (\varphi) v)$;

- une vitesse verticale vers le haut $(w)$ donne (4) une force vers l'Ouest $(-2 \Omega \cos (\varphi) w)$.

Les expressions des forces sont données par unité de masse, ce qui les apparente à des accélérations. Ces résultats s'appliquent à I'hémisphère Nord; dans I'hémisphère Sud, la latitude $\varphi$ devient négative, et dans ce cas il faut donc remplacer "vers le Sud » par « vers le Nord » après (1), et " vers l'Est » par "vers l'Ouest » après (3).

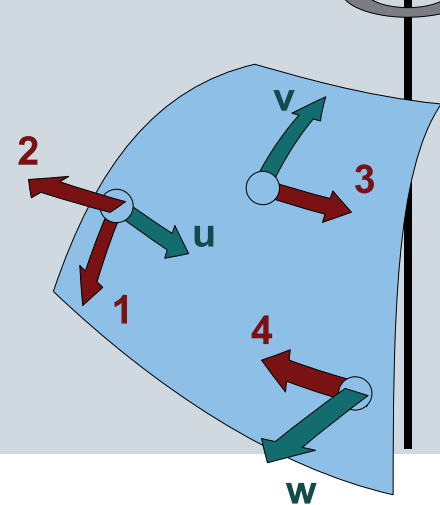


C'est dans le contexte de l'étude de l'influence des termes "traditionnels " (1) et (3) sur les marées que l'on a construit dans les années soixante une plaque tournante à Grenoble pour y étudier le chenal de la Manche, en vue de l'installation d'usines marémotrices de grande ampleur. La Manche $\mathrm{y}$ fut reproduite en similitude sur un disque de $13 \mathrm{~m}$ de diamètre. Cette plaque tournante, nommée "Plate-forme Coriolis ", sert aujourd'hui d'instrument européen dédié aux études fondamentales sur la mécanique des fluides en rotation (voir encadré, p. 21).

Qu'en est-il du rôle des termes non traditionnels, (2) et (4) ? C'est bien le terme (4) qui agit dans le problème de la chute libre, et est donc, d'un point de vue historique, le premier des quatre termes à avoir été étudié d'une manière profonde. La manifestation du terme (2) n'a été reconnue que beaucoup plus tard. Au début du $20^{\text {e }}$ siècle, au cours des campagnes de mesures sur les océans, on s'est aperçu que l'accélération de la pesanteur était systématiquement moins grande lorsque le bateau allait vers l'Est que lorsqu'il allait vers l'Ouest (typiquement d'un centième de pourcent). Ceci s'appelle l'effet "Eötvös", du nom du physicien hongrois qui en a fourni l'explication.

\section{Géostrophie}

En 1856, William Ferrel emprunta à la théorie des marées de Laplace les termes (1) et (3), et il les introduisit dans la météo-

\section{Littérature}

1 - A. Koyré, Chute des corps et mouvement de la terre: de Kepler à Newton, Vrin, Paris (1973).

2 - J.G. Hagen, La rotation de la terre, ses preuves mécaniques anciennes et nouvelles, Tipografia Poliglotta Vaticana, Roma (1911).

3 - H.L. Burstyn, "Early explanations of the role of the Earth's rotation in the circulation of the atmosphere and the ocean", Isis, 57 (1966) 167-187.

$4 \cdot \mathrm{M}$. Brillouin, Mémoires originaux sur la circulation générale de l'atmosphère, Carré et Naud, Paris (1900).

5 P.S. Laplace, Traité de Mécanique Céleste, Tome 1, Crapelet, Paris (1798).

$6 \bullet$ T. Gerkema, J.T.F. Zimmerman, L.R.M. Maas et H. van Haren, "Geophysical and astrophysical fluid dynamics beyond the traditional approximation", Rev. Geophys., 46, RG2004 (2008) 1-33.

7 - G. Coriolis, "Sur les équations du mouvement relatif des systèmes de corps ", J. Éc. Polytech. Paris, Cahier XXIV, Tome XV (1835) 142-154. rologie [4]. L'effet du terme (3) avait déjà été introduit par Hadley (au moins d'une façon qualitative), mais le terme (1) était alors nouveau dans ce domaine. La déviation due à ce dernier peut être comprise, explique Ferrel, de la manière suivante : une particule qui se déplace vers l'Est est soumise à une force centrifuge additionnelle (puisque sa propre célérité est complémentaire à celle due à la rotation de la Terre). Cette dernière est orientée vers l'extérieur dans le plan du cercle latitudinal. Sa décomposition en deux composantes, une méridionale et une radiale, donne ensuite les termes (1) et (2). Ferrel construisit aussi, à partir des données d'observation disponibles à l'époque, une représentation schématique de la circulation générale de l'atmosphère (fig. 2).

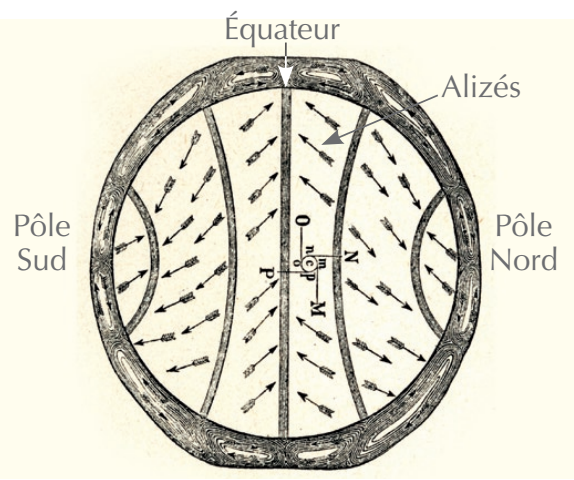

2. Représentation schématique de la circulation générale de l'atmosphère terrestre, établie par Ferrel en 1856. [Figure empruntée à Brillouin (1900)].

Dans chaque hémisphère il distingua trois bandes (les alizés, les vents d'Ouest aux latitudes moyennes, et les vents polaires) qui sont séparées par des lits de basse ou de haute pression. Ensuite, Ferrel supposa que ce gradient méridional de pression est en équilibre avec le terme (1), associé à un courant zonal $(u)$. Il introduisit ainsi la notion de ce que l'on appelle aujourd'hui l'équilibre géostrophique, selon lequel le vent suit les isobares au lieu d'être perpendiculaire à celles-ci comme on pourrait le penser.

\section{Le pendule de Foucault}

En 1851, Foucault fit sa fameuse expérience du pendule, dont il observa une rotation lente du plan vertical d'oscillation. Cette rotation est due aux termes (1) et (3), et sa période est donc inversement proportionnelle au sinus de la latitude. Dans les années qui suivirent, l'expérience fut répétée dans d'autres villes par de nombreux expérimentateurs ; une preuve directe de la rotation de la Terre frappait l'imagination, même si peu de

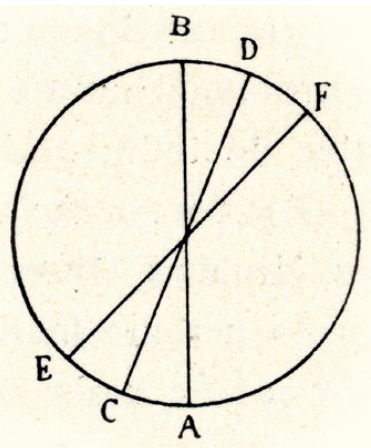

3. Le pendule de Foucault avant l'heure. Extrait des notes de Viviani (Accademia del Cimento, aux environs de 1660) : " Nous observâmes que tous les pendules à un seul fil dévient de leur plan [d'oscillation] et ce toujours dans le même sens, c'est-à-dire selon les lignes $A B, C D$, EF, etc. de la droite vers la gauche dans le sens du déplacement. » [Figure empruntée à Hagen (1911)].

personnes auraient encore douté de sa réalité. C'est bien là une tournure ironique de cette histoire, car l'effet avait en fait déjà été observé deux siècles auparavant, à une époque où l'existence même de la rotation de la Terre était l'objet d'un débat animé ; c'est ainsi que des membres de l'Accademia del Cimento firent mention d'une rotation en sens horaire du plan d'oscillation du pendule (fig. 3). Ils ne se sont alors pas rendu compte que la cause pouvait en être la rotation de la Terre. Pour eux, ce n'etait qu'un effet de perturbation; et plus tard ils fixèrent le pendule en deux points de manière à ce que sa rotation fut empêchée !

\section{Coriolis}

Les quatre termes (1) à (4), trouvés par Laplace, ne sont rien d'autre qu'une expression de ce qu'on appelera plus tard la "force de Coriolis ». Coriolis n'a donc pas été le premier à étudier la déflection due à «sa " force. Mais il fut bien le premier à associer d'une manière explicite cette déflection avec une force, qu'il appelait "force centrifuge composée " [7], et que l'on connait aujourd'hui comme "force de Coriolis ». D'ailleurs, son étude fut inspirée par des roues hydrauliques plutôt que par la rotation de la Terre.

Est-ce pour enrichir encore cette histoire que l'auteur même des équations qui portent aujourd'hui son nom, a su entourer son propre prénom d'une ambiguité sans pareille ? Les dictionnaires et encyclopédies mentionnent en proportion égale les prénoms Gustave et Gaspard, et son acte de décès Gaspard Gustave ; mais l'auteur, non sans ironie, signe sans manquer ses ouvrages du même éponyme : G. Coriolis ! 


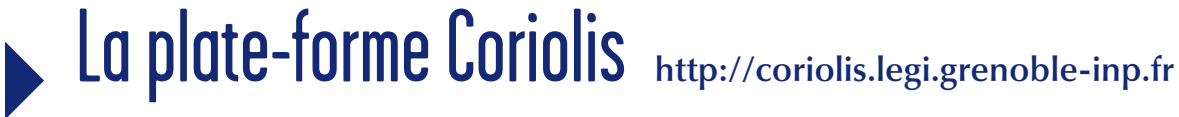

La plate-forme tournante Coriolis a été construite en 1960 à la demande d'EDF, qui voulait modéliser à l'échelle 1/50000 les courants et déplacements de sédiments créés par I'implantation d'une usine marémotrice dans la baie du Mont-Saint-Michel.

Cette machine a un diamètre de 13 mètres, une masse de 300 tonnes et elle peut atteindre une vitesse de rotation d'un tour toutes les 20 secondes. Il était ainsi possible de représenter les marées dans la Manche, en tenant compte des effets de la rotation terrestre et de la topographie des fonds marins dans la région. Ce dispositif a permis d'obtenir des résultats très proches de la réalité, même si l'aventure marémotrice a été ensuite abandonnée. Ces études ont cependant servi de base de comparaison pour le développement de programmes de modélisation numérique des marées, domaine qui a pris un essor considérable avec l'apparition de la cartographie des courants océaniques par satellite. C'est ainsi que le Laboratoire des Écoulements Géophysiques et Industriels (LEGI) à Grenoble s'est trouvé au cœur des développements nouveaux en océanographie opérationnelle.

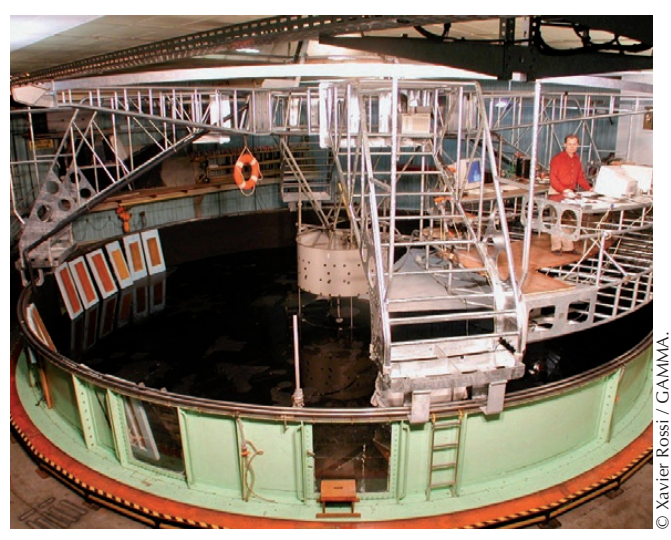

À la fin de l'étude du modèle physique de la Manche, les techniques de simulation numérique sont devenues assez efficaces pour prédire correctement les marées à l'échelle du globe, et ont rendu l'utilisation de la plate-forme à cette seule fin obsolète. Un crédit d'équipement du CNRS a alors permis, en 1985, de revenir vers des recherches plus fondamentales en dynamique des fluides géophysiques. Cette rénovation a été complétée par l'implantation de grandes cuves pour la préparation de solutions salines de différentes concentrations, ce qui permet, en plus, d'introduire une stratification de la densité des fluides en écoulement. Un portique d'instrumentation a également été mis en place. Ces équipements ont été accompagnés de développements innovants en mesure de champs de vitesse par imagerie laser.

Aujourd'hui, des chercheurs du monde entier viennent réaliser sur cette installation des expériences à grande échelle sur les fluides stratifiés en rotation (voir image de couverture et photo p. 18).

Louis Gostiaux 